\title{
Capacitive readout and gating of superconducting single photon detectors
}

\author{
Hatim Azzouz, ${ }^{1,2, a)}$ Reinier W. Heeres, ${ }^{2, a)}$ Sander N. Dorenbos, ${ }^{2}$ Raymond N. Schouten, ${ }^{2}$ \\ and Valery Zwiller² \\ ${ }^{1}$ Physics Department, Stockholm University, SE 10691 Stockholm, Sweden \\ ${ }^{2}$ Kavli Institute of Nanoscience, Delft University of Technology, P.O. Box 5046, 2600 GA Delft, The Netherlands
}

(Received 4 October 2012; accepted 26 April 2013; published online 22 May 2013)

\begin{abstract}
We propose and develop a readout scheme for superconducting single-photon detectors based on an integrated circuit, relaxing the need for large bandwidth amplification and resulting in voltage steps proportional to the number of detected photons. We also demonstrate time gating, to filter scattered light in time and reduce dark counts. This could lead to a higher signal-to-noise ratio. The gate pulse is generated on the detection of a photon created by a spontaneous parametric downconversion source, heralding the presence of a second photon. These two schemes could find applications within advanced multi-array imaging detection systems. (C) 2013 AIP Publishing LLC. [http://dx.doi.org/10.1063/1.4805055]
\end{abstract}

\section{INTRODUCTION}

Superconducting single-photon detectors (SSPDs) ${ }^{1}$ are becoming the most promising alternative to conventional single photon detectors. Their high saturation count rates, small timing jitter, broad wavelength detection range, and low dark count rates $^{2}$ make them promising candidates in applications such as quantum key distribution. ${ }^{3-6}$ Recent work has shown efficiency improvements at telecom wavelengths by employing cavities. ${ }^{7,8}$ Medical imaging ${ }^{9}$ and biophotonics ${ }^{10}$ are among envisioned applications. ${ }^{11}$ Detection efficiency increases with current bias, along with dark count rates. In some implementations, bias current must be limited to avoid large dark count rates. Time gating of SSPDs would allow high detection efficiency over a time range without an associated large dark count rate, much like the technique employed for avalanche photodiodes. Here we introduce a fast time gating technique. This together with the presented capacitive readout scheme will improve a number of applications including imaging based on SSPD arrays and biomedical applications such as fluorescence lifetime measurements. ${ }^{12}$

Details of our device fabrication are described elsewhere. ${ }^{13}$ To summarize, the detector was fabricated by standard nanofabrication techniques using electron beam lithography and reactive ion etching on an oxidized silicon substrate. The SSPD is a $500 \mu \mathrm{m}$ long NbTiN nanowire, $100 \mathrm{~nm}$ wide, and about $4 \mathrm{~nm}$ thick meandering over $10 \times 10 \mu \mathrm{m}$ with a filling factor of $50 \%$.

\section{CAPACITIVE READOUT SCHEME}

Traditional SSPD operation involves counting voltage pulses triggered by single photon detection events. This requires high gain amplifiers with large bandwidth $(54 \mathrm{~dB}$, $\sim 1 \mathrm{GHz}$ ) and pulse counting electronics. We replaced the high-frequency amplification stage by a capacitor functioning as an integrating element to simplify the operation. The use of a capacitor requires less power in operation. Most im-

a) H. Azzouz and R. W. Heeres contributed equally to this work. portantly, this also allows the building of SSPD array systems by using low-temperature multiplexers ${ }^{14-16}$ and sequentially reading out the capacitors corresponding to individual pixels. This in turn allows all photons to be detected and their position to be determined. We demonstrate experimentally that the readout voltage is quantized in steps and can be used for photon counting.

Prior to the experiment, a simulation of the capacitor voltage readout was performed. The electronic model used in the simulation (Micro-cap 9) is shown schematically in Figure 1(a). The SSPD was modeled as a switch in parallel with a $6 \mathrm{k} \Omega$ resistor $^{17}$ and the system was biased with a DC current $I_{D C}$ ( $V_{b}$ over a $100 \mathrm{k} \Omega$ resistor). The fast switch simulates the operation of photon detection. Having the switch closed corresponds to the superconducting state of the SSPD, whereas an open switch corresponds to the resistive state when a photon is absorbed. To simulate a detection event, the switch opens for $0.3 \mathrm{~ns}$ and the repetition rate of the switching operation is set to $16 \mathrm{MHz}$, simulating detected events separated by $62.5 \mathrm{~ns}$. The model values are based on measurements and literature. The SSPD kinetic inductance $L_{k}$, which limits the detector count rate, is determined from the detection pulse shape $\tau_{L R}=\frac{L_{k}}{50 \Omega} .{ }^{18}$ The detection pulse is shown in Figure 1(b). The measured value of $\tau_{L R}$ is $\sim 5 \mathrm{~ns}$, implying a kinetic inductance of $250 \mathrm{nH}$. A capacitor of $1 \mathrm{nF}$, which constitutes the main element in this scheme, was introduced in the circuit. This gives an RC-time $\tau_{R C}$ of $(50 \Omega$ $\left.+R_{d}\right) C \mathrm{~s}$, where $50 \Omega$ corresponds to the termination resistance to match the coaxial line impedance and $R_{d}$ is the discharge resistor $(\sim 10 \Omega)$, which sets the time constant. The readout voltage was probed at the node labeled "readout." According to the simulation, for $n$ detected photon, $n$ steps will sequentially be formed on top of the capacitor voltage readout measurement indicating $n$ detected photons. In principle, decreasing the capacitor value down to orders of picoFarads results in an increase of the readout amplitude to the order of millivolts. However, to get a reasonable RC-time constant of $\sim 60$ ns the capacitance was chosen to be $1 \mathrm{nF}$ (with, in this case, a resistance $R$ of $60 \Omega$ ). 
(a)

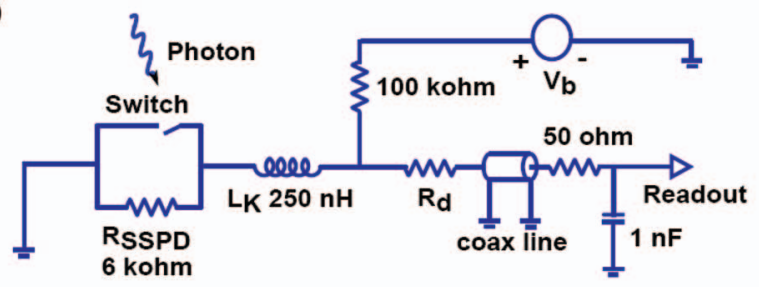

(b)

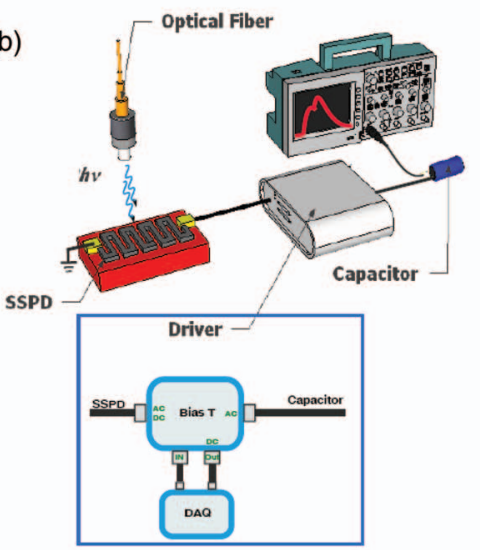

(c)

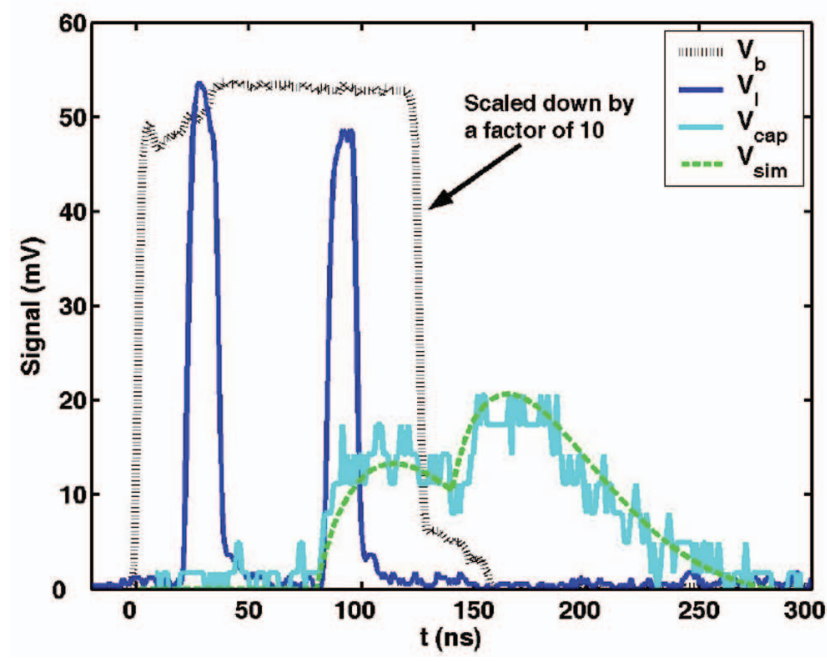

FIG. 1. (a) Simulation diagram and (b) experimental setup. The pulse is a recorded detection of a striking photon without a capacitor. (c) The bias voltage $\left(V_{b}\right)$, voltage pulses driving the laser diode $\left(V_{l}\right)$, a single shot capacitor voltage readout measurement $\left(V_{C a p}\right)$ and simulation result $\left(V_{\text {sim }}\right)$. The bias voltage is scaled down by a factor of 10 .

The measurements were performed in a liquid helium bath cryostat at $4.2 \mathrm{~K}$. A $633 \mathrm{~nm}$ pulsed laser diode was used for optical excitation and was focused onto the detector by a coupling lens (NA $0.25, \mathrm{f}=11 \mathrm{~mm}$ ). The SSPD was mounted on a circuit board and connected through a coaxial cable to the electronics driver box as shown in Figure 1(b). The driver box, composed of a homemade Bias $\mathrm{T}(1 \mathrm{MHz}-5 \mathrm{GHz})$, was connected to the detector through the $\mathrm{AC}+\mathrm{DC}$ port and through the DC component to a computer-controlled constant current source. A biasing voltage was applied by a data acquisition (NI DAQPad-6015) box through the Bias T and converted to current by a $500 \mathrm{k} \Omega$ resistor before continuing to the SSPD. The capacitor was connected through the AC port and then to a $44 \mathrm{~dB}$ amplifier (ZX60-33LN) before monitoring on the os- cilloscope $1 \mathrm{GHz}$ oscilloscope (LeCroy, LC574AM). Assuming an SSPD pulse length of $\tau_{L R} \sim 5 \mathrm{~ns}$, we expect the capacitor readout voltage prior to amplification to be in the order of tens of microvolts $\left(V=\frac{I t}{C}\right.$, where $\left.I \sim 10 \mu \mathrm{A}\right)$.

Figure 1(c) shows both experimental and simulation results obtained for the capacitor readout. Within the $120 \mathrm{~ns}$ time window that the detector was biased with $\sim 10 \mu \mathrm{A}$, two laser pulses were applied to the SSPD with a repetition rate of $16 \mathrm{MHz}$. A single shot readout of the capacitor voltage shows the expected integrating behaviour and contains two distinct peaks separated by around $60 \mathrm{~ns}$, corresponding to two detection events. The delay between the laser pulses and detected events was set by the length of the coaxial cables and the optical fiber. The simulated curve $V_{\text {sim }}$ is in good agreement with the measured capacitor readout $V_{C a p}$. Most of the recorded events $(\sim 75 \%)$ showed only one peak on the capacitor readout curve, indicating a single photon detection. A fraction of the recorded events $(\sim 20 \%)$ showed a double peak thereby enabling a distinction between 1 and 2 detection events based on the readout amplitude.

\section{TIME GATING}

Gating can increase the signal to noise ratio by enabling application of a larger current (and hence a higher detection efficiency) during a short time window when a photon is expected, without suffering from large dark noise when no photon is expected. Figure 2(a) shows the setup used for gating our detector. Here, a single mode fiber was pigtailed to the SSPD providing the optical illumination. The pigtailed detector was mounted on a dip-stick and immersed in liquid helium. It was electrically connected as shown in Figure 1(b), but instead of sending the output signal to the capacitor it was amplified directly by $\sim 54 \mathrm{~dB}$ over a bandwidth of $\sim 1 \mathrm{GHz}$ and sent to an oscilloscope. A constant DC bias current of $\sim 4 \mu \mathrm{A}$, about $80 \%$ of the critical current, was applied.

The optical illumination in the schematic of Figure 2(a) was provided by a spontaneous parametric down-conversion (SPDC) source. A continuous wave $532 \mathrm{~nm}$ pump laser drove a collinear SPDC process in a $2 \mathrm{~cm}$ long KTP crystal cut for degenerate type-II phase-matching. After passing through the crystal, a polarizing beam-splitter separated the generated photon pairs (coherence time $2 \mathrm{ps}$ ), and the individual correlated $1064 \mathrm{~nm}$ photons were collected in single-mode fibers. One of these fibers was connected to an Avalanche Photodiode Detector (APD)(PerkinElmer, SPCM-AQR-WX-FC), a detection event by this detector heralds a photon in the other fiber, connected to the pigtailed SSPD. The APD signal was converted to a proper gating pulse with a pulse stretcher (to create a $50 \mathrm{~ns}$ wide TTL signal), a $80 \mathrm{~dB}$ attenuator, and a $25 \mathrm{MHz}$ low pass filter with $\sim 13 \mathrm{~ns}$ rise time. The filter blocks the direct cross-talk between the gating pulse and the ACcoupled high-frequency pulse detection electronics. The pulse was then applied across a $500 \Omega$ resistor to add it to the DC bias current. The processing of the APD signal caused a delay of $\sim 10 \mathrm{~ns}$. This gating pulse current was carefully adjusted, when the high SNR is achieved. This was achieved at about $1 \mu \mathrm{A}$. Although the exact total bias current is unknown at $80 \%$ DC bias ratio, we estimated the gating pulse current to 


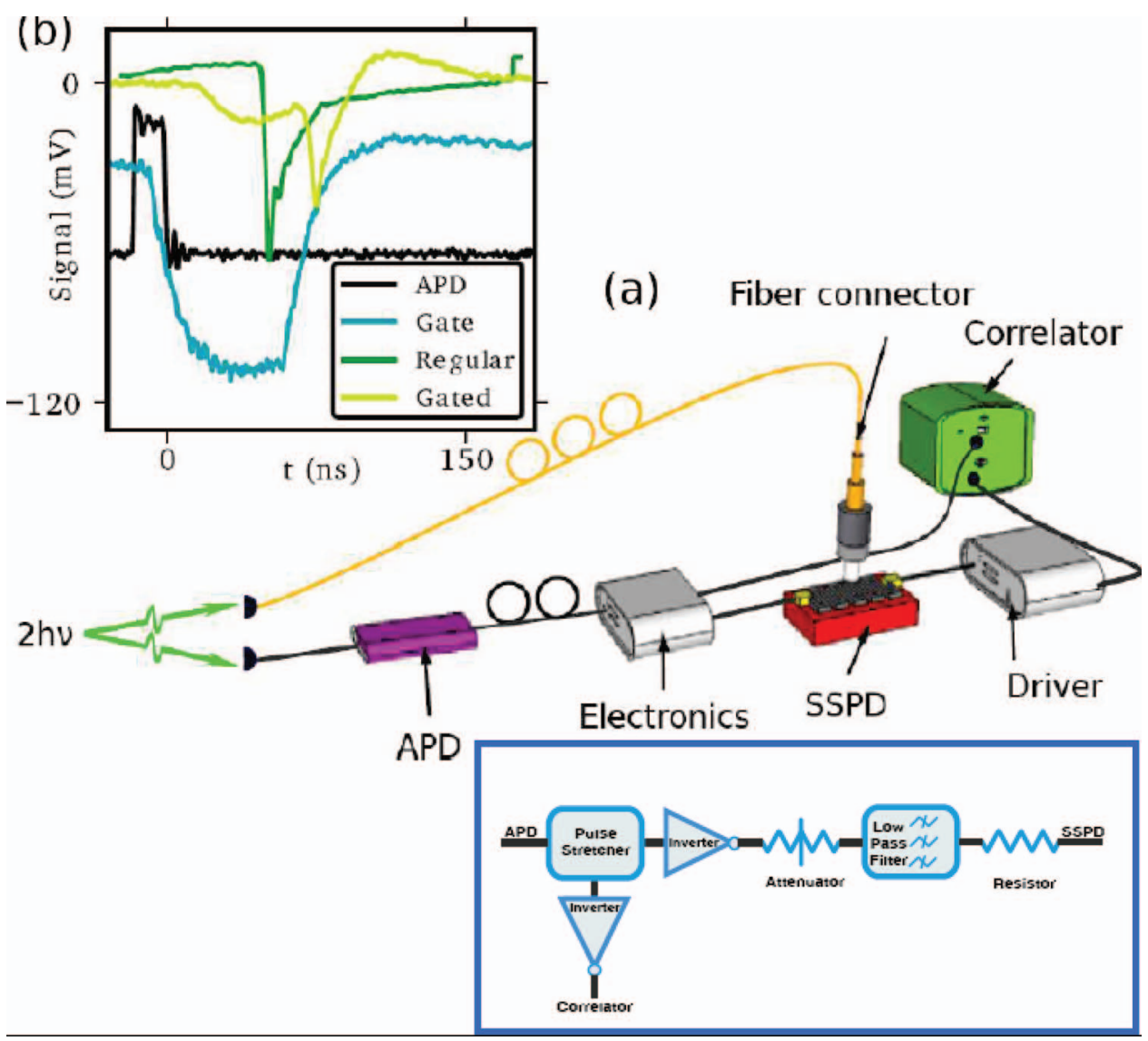

FIG. 2. (a) Experimental setup for gating. The electrical readout is represented by the driver box. The electronic circuit box is composed of a pulse stretcher ( $50 \mathrm{~ns}$ wide TTL signal) connected in series to an inverter (PicoQuant SIA 400, In TTL, Out $\sim 300 \mathrm{mV}$ ), an $80 \mathrm{~dB}$ attenuator (Mini Circuits ZX73-2500), a low pass filter (Mini Circuits, BLP25) and a $500 \Omega$ resistor. (b) Gating and measured signals. The APD detection pulse (APD), scaled by a factor 0.02 , was reshaped by the electronics box into a $50 \mathrm{~ns}$ inverted gate pulse (Gate). The gate pulse is scaled up by a factor of 100. A regular detection event without gating the SSPD (Regular) and an event occurring when a gate pulse is applied (Gated).

correspond to a fraction of $20 \%$ of the applied bias current. The main DC component was provided by the driver $\left(I_{A P D} \ll\right.$ $\left.I_{\text {Bias }}, I_{A P D}+I_{\text {Bias }}=I_{\text {TotalBias }}\right)$. However, $I_{\text {Bias }}$ could be kept smaller than usual, resulting in very low or no dark counts when the detector was not gated on. The involved signals, APD pulse, gate pulse, regular detection event, and gated detection event, are shown in Figure 2(b).
To measure the correlation between photons detected by the SSPD and the APD, both output signals were sent to a time-correlated single-photon counter module (PicoHarp 300 , resolution set to $256 \mathrm{ps}$ ). Figure 3 compares the event detection falling within and outside the gating window. Figure 3(a) shows the measurement where the heralded photons were delayed by successively adding $1 \mathrm{~m}$ optical fiber
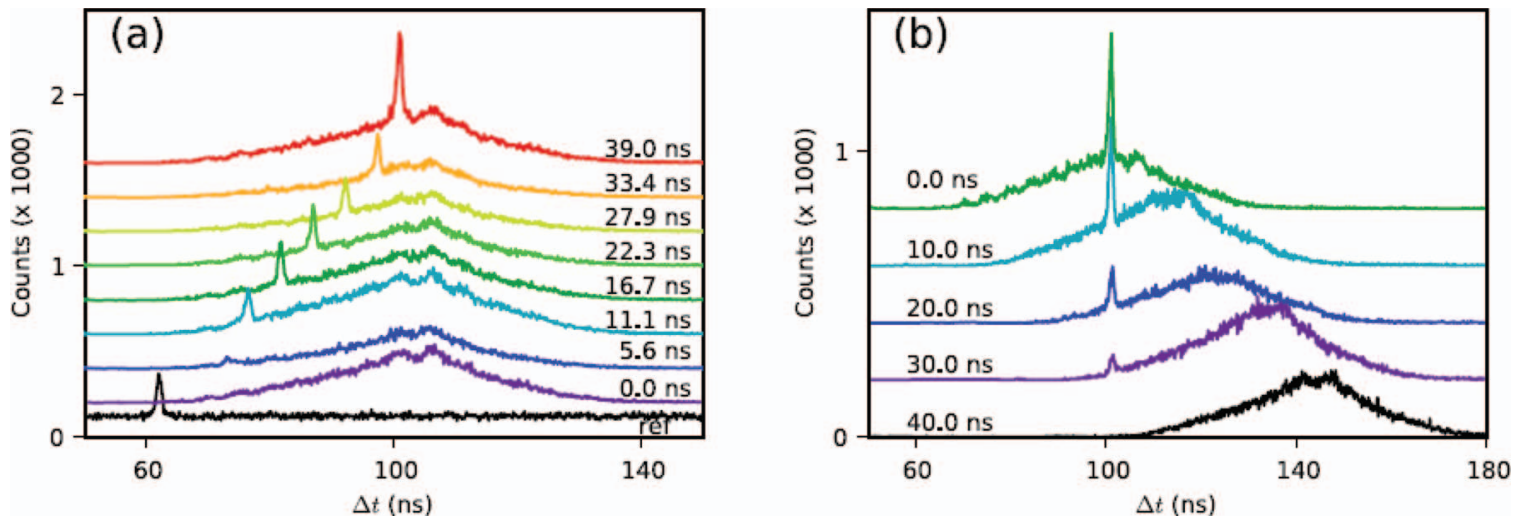

FIG. 3. Gated operation of the SSPD. (a) Detection events as a function of optical delay of the heralded photon. The reference shows detection events for a $7 \mathrm{~m}$ fiber optical delay without gating, i.e., without the use of APD. Therefore, the gating envelope of $50 \mathrm{~ns}$ is non-occurring and the reference curve is flat. (b) Detection events as a function of gate pulse delay. When the heralded photon falls outside the gate window (lowest trace) there are no correlated detection events. 
patches. Figure 3(b) shows the results when the gate pulse was delayed by sequentially adding $1 \mathrm{~m}$ extra length to the coax line. The correlated photons show up as a sharp peak, indicating that detection of a photon by the APD heralds the presence of another photon at the SSPD. For the case where the heralded photons fall outside the gate window, no peaks (correlated detection events) are recorded. Since the timing jitter of the APD and SSPD are much larger than the coherence time of the photon pairs, the width of the peak is given by the jitter. The envelope of the $\sim 50 \mathrm{~ns}$ wide curve, where detection events occur, is caused by both background light and dark counts. It gives an indication of the shape of the gating pulse applied to the SSPD, and thus a good hint for the efficiency of detecting a photon.

\section{CONCLUSION}

In conclusion, we have demonstrated the implementation of a capacitor-based voltage readout, allowing us to resolve the number of incoming photons within the RC-time of the capacitor and relax amplification requirements. This was simulated using a circuit model with two photon pulses and confirmed by measurements. For an SSPD array using lowtemperature multiplexing readout, this will allow all photons to be detected and their position to be determined, resulting in a single-photon imaging detector with low noise that is sensitive over a broad wavelength range. Furthermore, we have shown that it is possible to gate an SSPD to provide some of the time-information that is lost when using capacitive readout. Further work is ongoing on improving and increasing the detection efficiency in small time-windows when photons are expected, without suffering from dark counts outside this window, and with the hope of eventually fabricating arrays of these detectors.

\section{ACKNOWLEDGMENTS}

The authors would like to thank R. H. Hadfield for fiber pigtailing the detector. This work is supported financially by the Swedish Foundation for International Cooperation in Research and Higher Education (STINT) and by the Netherlands Organisation for Scientific Research (NWO/FOM).

${ }^{1}$ G. Gol'tsman, O. Okunev, G. Chulkova, A. Lipatov, A. Semenov, K. Smirnov, B. Voronov, A. Dzardanov, C. Williams, and R. Sobolewski, Appl. Phys. Lett. 79, 705 (2001).

${ }^{2}$ G. Gol'Tsman, O. Minaeva, A. Korneev, M. Tarkhov, I. Rubtsova, A. Divochiy, I. Milostnaya, G. Chulkova, N. Kaurova, B. Voronov et al., IEEE Trans. Appl. Supercond. 17, 246 (2007).

${ }^{3}$ H. Takesue, S. Nam, Q. Zhang, R. H. Hadfield, T. Honjo, K. Tamaki, and Y. Yamamoto, Nat. Photonics 1, 343-348 (2007).

${ }^{4}$ R. H. Hadfield, J. L. Habif, J. Schlafer, R. E. Schwall, and S. W. Nam, Appl. Phys. Lett. 89, 241129 (2006).

${ }^{5}$ Z. Wang, S. Miki, and M. Fujiwara, IEEE J. Sel. Top. Quantum Electron. 15, 1741 (2009).

${ }^{6}$ R. H. Hadfield, Nat. Photonics 3, 696 (2009).

${ }^{7}$ X. Hu, E. A. Dauler, R. J. Molnar, and K. K. Berggren, Opt. Express 19, 17 (2011).

${ }^{8}$ M. Tanner, C. Natarajan, V. Pottapenjara, J. O'Connor, R. Warburton, R. Hadfield, B. Baek, S. Nam, S. N. Dorenbos, E. Ureña, T. Zijlstra, T. Klapwijk, and V. Zwiller, Appl. Phys. Lett. 96, 221109 (2010).

${ }^{9}$ D. J. Hall, J. C. Hebden, and D. T. Delpy, Appl. Opt. 36, 7270 (1997).

${ }^{10}$ P. Schwille, Cell Biochem. Biophys. 34, 383 (2001).

${ }^{11}$ G. Buller and R. Collins, Meas. Sci. Technol. 21, 012002 (2010).

${ }^{12}$ M. J. Stevens, R. H. Hadfield, R. E. Schwall, S. W. Nam, R. P. Mirin, and J. A. Gupta, Appl. Phys. Lett. 89, 031109 (2006).

${ }^{13}$ S. Dorenbos, E. Reiger, U. Perinetti, V. Zwiller, T. Zijlstra, and T. Klapwijk, Appl. Phys. Lett. 93, 131101 (2008).

${ }^{14}$ L. M. Reyneri, Cryogenics 29, 540 (1989).

${ }^{15}$ J. Yoon, J. Clarke, J. M. Gildemeister, A. T. Lee, M. J. Myers et al., Appl. Phys. Lett. 78, 371 (2001)

${ }^{16}$ A. Benoit, S. J. C. Yates, E. Grémion, P. Camus, T. Durand, C. Hoffmann, S. Leclerq, A. Monfardini, A. Cavanna, B. Etienne, U. Gennser, and Y. Jin, J. Low Temp. Phys. 151, 940 (2008).

${ }^{17}$ J. Yang, A. Kerman, E. Dauler, V. Anant, K. Rosfjord, and K. Berggren, IEEE Trans. Appl. Supercond. 17, 581 (2007).

${ }^{18}$ A. Kerman, E. Dauler, W. Keicher, J. Yang, K. Berggren, G. Gol'tsman, and B. Voronov, Appl. Phys. Lett. 88, 111116 (2006). 